\title{
Size Effect in Paris Law and Fatigue Lifetimes for Quasibrittle Materials: Modified Theory, Experiments and Micro-Modeling
}

\author{
Kedar Kirane $^{\mathrm{a}}$, Zdeněk P. Bažant ${ }^{\mathrm{a}, *}$ \\ ${ }^{a}$ Department of Civil and Environmental Engineering, Northwestern University, 2145 Sheridan Road, Evanston, Il. \\ 60208 USA.
}

\begin{abstract}
Unlike brittle materials, quasibrittle materials exhibit a structure size effect on the fatigue crack growth rate, particularly on the Paris law coefficient (prefactor). This size effect is strong for specimens not much larger than the dominant material inhomogeneities (or aggregate sizes in concrete), and vanishes for very large structures. It can be quantified by a size adjustment of Paris law which is similar to the size effect law for monotonic loading. But the transitional size $D_{0 c}$ at which the transition is centered is not the same. Previous experiments aimed at quantitative analysis of this size effect involved only one or a few specimens per size. Thus the huge scatter, inevitable in fatigue tests, distorted the estimates of $D_{0 c}$ and, thereby, also of the size of the cyclic fracture process zone (FPZ), to which $D_{0 c}$ is proportional. Here, more reliable estimates of $D_{0 c}$ and the cyclic FPZ size are obtained by conducting, on concrete, multiple fatigue tests per size and taking the average. Furthermore, these length characteristics are also estimated numerically using the latest version of the microplane constitutive damage model for concrete (model M7), extended to quasibrittle fatigue. It is conclusively shown that the cyclic FPZ is smaller than the monotonic FPZ. Further, the numerical simulations of the cyclic deformations within the FPZ reveal that the $D_{0 c}$ obtained from the previous form of the size-adjusted Paris law is not proportional to the cyclic FPZ size. A new form is proposed and verified by updated dimensional analysis. It involves the transitional sizes for both the monotonic and cyclic size effects and is seen to yield values of $D_{0 c}$ that are proportional to the cyclic FPZ size. The ensuing size effect on fatigue lifetimes is simulated using both the previous and new forms. Both forms are found to predict a non-monotonic size effect on the lifetimes, initially decreasing and, after a minimum, eventually increasing with increasing size. It is also shown that, similar to Paris law for brittle fatigue, an extension to the fatigue threshold at nearly vanishing amplitude is impossible because of a transition to Charles-Evans law for static fatigue. Finally, a possible ramification to fatigue of micrometer-scale metallic devices is pointed out.
\end{abstract}

Keywords: Scaling of fatigue, Fatigue crack growth, Fatigue lifetime, Quasibrittle materials, Numerical simulation, Dimensional analysis, Cyclic fracture process zone, Statistical scatter

\section{Introduction}

The mechanics of fracture and fatigue is of three kinds: brittle, brittle-ductile and quasibrittle. In brittle fracture mechanics, conceived by Griffith in 1921, the fracture process zone (FPZ) can

${ }^{*}$ Corresponding author. Tel.: +1-847-491-4025; fax: +1-847-491-4011.

Email address: z-bazant@northwestern.edu (Zdeněk P. Bažant)

(C) 2015. This manuscript version is made available under the Elsevier user license http://www.elsevier.com/open-access/userlicense/1.0/ 
be considered to be a point and the specimen or structure follows linear elastic fracture mechanics (LEFM). In brittle-ductile fracture, most of the FPZ is plastic although the FPZ can still be treated almost as point embedded in the plastic zone. In quasibrittle fracture [1, e.g.], most of the nonlinear zone surrounding the crack tip represents the FPZ and undergoes progressive softening damage. The fact that the FPZ size in quasibrittle materials is not negligible compared to structural dimensions gives rise to transitional size effects. The size effect in quasibrittle fatigue was first demonstrated in [11; 12]. Recently it was carefully analyzed for rock by Le et al. [13]. Their work is here extended to concrete and the use of microplane model makes it possible to resolve the inelastic phenomena within the FPZ governing the fatigue crack growth.

Fatigue crack growth in a wide variety of brittle materials is described well by the famous Paris (or Paris-Ergodan) law [14], which relates the rate of crack growth per cycle $\mathrm{d} a / \mathrm{d} N$ to the amplitude of the stress intensity factor $\Delta K$ via a power law, and is expressed as,

$$
\frac{\mathrm{d} a}{\mathrm{~d} N}=C(\Delta K)^{m}
$$

where $C$ and $m$ are material parameters dependent on the load ratio $R$, the environment, etc. [15]. This law was shown to be applicable to quasibrittle materials as well $[11 ; 12 ; 13]$. These heterogeneous materials with brittle constituents include concrete, as the archetypical case, many rocks, tough ceramics, fiber composites (and even metals at the micrometer scale). Quasibrittle fatigue crack growth is characterized by a large FPZ that is non-negligible compared to structural dimensions, and its localization is limited by a finite material characteristic length. This engenders a structural size effect on the fatigue crack growth, causing a size effect in Paris law and consequently on the fatigue lifetimes. This has important implications for the fatigue design and lifetime safety factors of quasi-brittle structures in a variety of civil, aerospace, energy and even semi-conductor applications.

${ }^{c 1}$ The topic of scaling in Paris law has been studied for metals by many researchers; e.g., $\left[2 ; 3 ; \overline{4 ; 5 ; 6 ; 7]^{\mathrm{c} 2} \text { but mainly from an experimental standpoint. Some researchers have also analyzed }}\right.$ this problem using dimensional analysis $[8 ; 9 ; 10] .{ }^{\mathrm{c} 3}$ But very few studies aimed at a truly quantitative understanding of this size effect, which include $[11 ; 12 ; 13] .{ }^{4}$ These led to the development of a 'size adjusted' Paris law. ${ }^{c 5}$ It was also inferred in these studies that the size of the cyclic FPZ is different from the monotonic one. However there was no general consensus about how different it is. The reason was that the aforementioned studies failed to take into account the experimental scatter, which is huge for fatigue. Further, they did not study the size effect in lifetime. Thus, the goals of this study are:

1. Experimental verification of the existing size adjusted Paris law, specifically of the estimates of the transitional size $D_{0 c}$ and the cyclic FPZ size. The fatigue experiments considered were performed on geometrically scaled concrete beams of multiple sizes, with multiple specimen per size. The average response of several specimens will be analyzed so as to minimize the influence of scatter.

\footnotetext{
c1 Text added.

c2 Text added.

c3 Text added.

c4 Text added.

c5 Previous studies, aimed at a quantitative understanding of this size effect led to the development of a 'size adjusted' Paris law.
} 
2. Numerical verification and FPZ resolution, based on the recently developed damage model for quasibrittle fatigue [16], which is a refinement of microplane model M7. The numerical approach is expected to predict the mean behavior with reduced scatter. Furthermore, it makes possible accurate determination of the physical FPZ sizes, which provides additional means for verification.

3. Size Effect Extension to fatigue lifetimes, resulting from the size effect on Paris law.

\section{Existing form of the size adjusted Paris law}

The size effect on structural strength of brittle and quasibrittle materials is by now well understood $[1 ; 17]$. In the case of purely brittle fracture, the LEFM holds. The scaling of structural strength $\sigma_{N}$ is self similar, described by a power law $\left(\sigma_{N} \propto D^{-1 / 2}\right)$. However for quasibrittle fracture, the structural strength scaling exhibits incomplete self-similarity, necessitating the introduction of a characteristic length scale. For geometrically scaled structures with a pre-existing crack or a notch, this size effect is well described by Bažant's size effect law [1],

$$
\sigma_{N}=B f_{t}^{\prime}\left(1+\frac{D}{D_{0 m}}\right)^{-1 / 2}
$$

where $\sigma_{N}$ is the nominal structure strength, $D$ is the structure size, $B$ is a material parameter, $f_{t}^{\prime}$ is a measure of the material strength, and $D_{0 m}$ is the transitional size which delineates ductile and brittle failures under monotonic loading fracture and depends on the material characteristic length as well as structural geometry. Based on asymptotic expansion of the deviations from LEFM,

$$
B=\frac{1}{f_{t}^{\prime}} \sqrt{\frac{E^{\prime} G_{f}}{g^{\prime}\left(\alpha_{0}\right) c_{f m}}} ; \quad D_{0 m}=c_{f m} \frac{g^{\prime}\left(\alpha_{0}\right)}{g\left(\alpha_{0}\right)}
$$

where $c_{f m} \approx$ half the FPZ length during monotonic fracture, $\alpha_{0}=a_{0} / D$, the relative initial crack length, $g\left(\alpha_{0}\right)=k^{2}\left(\alpha_{0}\right)=$ dimensionless energy release rate, $k\left(\alpha_{0}\right)=$ dimensionless stress intensity factor, which accounts for geometry effects, $g^{\prime}\left(\alpha_{0}\right)=\mathrm{d} g(\alpha) /\left.\mathrm{d} \alpha\right|_{\alpha=\alpha_{0}}$; for plane strain $E^{\prime}=E /\left(1-\nu^{2}\right), E=$ Young's modulus, $\nu=$ Poisson ratio, $G_{f}=$ fracture energy (under monotonic conditions).

The size effect law has been proven to describe the scaling of monotonic strength in a wide variety of quasibrittle materials [1].

The size effect on Paris law for quasibrittle materials was first experimentally identified in [11] and [12] by fatigue tests of geometrically scaled three-point bend beams of normal and high strength concretes (NSC and HSC). It was found that the Paris law plots $\log (\mathrm{d} a / \mathrm{d} N)$ vs. $\log (\Delta K)$ of similar beams of different sizes are not coincident, but spaced apart and roughly parallel in a log-log plot, implying a size effect in the Paris law coefficient $C$ but not the exponent $m$. To express the Paris law as a material property, independent of structure size, a size adjusted Paris law was proposed [11] in the form

$$
\frac{\mathrm{d} a}{\mathrm{~d} N}=C\left(\frac{\Delta K}{K_{I C}}\right)^{m} \text { where } K_{I C}=K_{I F}\left(1+\frac{D_{0 m}}{D}\right)^{-1 / 2}
$$

which follows from Eqs. 2 and 3 ; here $K_{I C}=$ size dependent fracture toughness, such that $\lim _{D \rightarrow \infty} K_{I C}=K_{I F}$. The purpose was to collapse the Paris law plots for different sizes into a unified plot. 
However, using the $D_{0 m}$ value from the monotonic size effect law would not unify the plots. Therefore, $D_{0 m}$ in Eq. (3) was replaced by a similar expression for the transitional size for fatigue, $D_{0 c}=c_{f c} g^{\prime}\left(\alpha_{0}\right) / g\left(\alpha_{0}\right)$ in which $c_{f c} \approx$ half the size of the cyclic FPZ. The value of $D_{0 c}$ was determined iteratively, such that it would cause the three distinct Paris law plots for different sizes to merge into one. This confirms Rice's proposition [18] that the cyclic and monotonic FPZs must be different in size. Further it reveals approximate equality of the ratio, $R_{f}$, of the cyclic and monotonic FPZ sizes, $L_{f c}$ and $L_{f m}$ to the ratios of the transitional sizes and of the approximate FPZ half-sizes, i.e.,

$$
R_{f}=\frac{D_{0 c}}{D_{0 m}}=\frac{c_{f c}}{c_{f m}} \approx \frac{L_{f c}}{L_{f m}}
$$

The ratio $R_{f}$ was reported as 10 in [11] but later discounted, due an error found in copying the compliance equation from the handbook. Further, $R_{f}$ was reported in [12] to be $\approx 1.5$ but after a revised fitting of the monotonic size effect law, it is now found to be $\approx 0.9$. So, although it was originally inferred that the cyclic FPZ would be more elongated than the monotonic one, this inference now comes under question.

Recently, this fatigue size effect was studied by Le et al. [13] for sandstone, a rock that is also quasibrittle. They realized that since $K_{I C}$ is the monotonic fracture toughness, its scaling cannot involve $D_{0 c}$. So, they introduced a new physical quantity, viz. the fatigue fracture energy $U_{c}$, which is the energy required to propagate a fatigue crack of unit width by a unit length. The size effect in $U_{c}$ involved $D_{0 c}$ and was expressed as [13],

$$
U_{c}=U_{c, \infty} \frac{D}{D+D_{0 c}}
$$

where $U_{c, \infty}$ is the fatigue fracture energy for $D \rightarrow \infty$. Rather than normalizing $\Delta K$ by $K_{I C}$, Le et al. introduced the dimensionless parameter $\Delta K^{2} / E U_{c}$ and conducted dimensional analysis to reach the following size-adjusted Paris law:

$$
\frac{\mathrm{d} a}{\mathrm{~d} N}=C\left(1+\frac{D_{0 c}}{D}\right)^{m / 2}(\Delta K)^{m}=C\left(\Delta K_{D}\right)^{m}
$$

where $\Delta K_{D}=\Delta K\left(1+D_{0 c} / D\right)^{1 / 2}=$ size adjusted $\Delta K$. Interestingly, they arrived at the same form as in [12] but by using more elegant arguments. By applying the aforementioned iterative process, they determined $D_{0 c}$ leading to $R_{f}=1.5$. This indicates a more elongated cyclic FPZ, which differs from the deduction in [12].

Unfortunately, all the foregoing experimental deductions of $D_{0 c}$ and $R_{f}$ were impaired by large experimental scatter which, in the case of lifetime, can span even two orders of magnitude. This

causes a big scatter in the Paris law coefficient $C$, which in turn is projected into $D_{0 c}$ and $R_{f}$. Thus it appears that the previous estimates are not reliable. More reliable results may be obtained by testing multiple specimens per size, and considering the average response per size. The results of such tests are presented next.

\section{Experiments on size effect in Paris law}

\subsection{Description of test set up}

Geometrically scaled concrete beams of three different sizes were tested under cyclic loading conditions, in a three point bend configuration. To take advantage of a preceding extensive experimental study of concrete fracture conducted at Northwestern University [19], which provided 
thorough information on all the main material characteristics, the leftover specimens from that study were used. In that study, 140 fracture test specimens were cast from the same batch of ready-mixed concrete, which ensured an unusually low coefficient of variation of scatter of material properties (see [19]), and were tested under monotonic loading. The present test specimens were prepared by sawing from the undamaged parts of the broken halves of the large three-point bend specimens used previously for monotonic strength and fracture tests [19] (a diamond band-saw with a $2 \mathrm{~mm}$ thick blade was used). The age of the concrete during strength and monotonic fracture testing was one year [19], while during the present fatigue testing, about two years. During the time gap, the broken halves of the beams were stored in a curing room with $100 \%$ relative humidity so as to avoid any drying effects. Since the chemical reactions of cement hydration at room temperature are generally believed to terminate within about one year, the elastic properties and strength of the concrete used could not have changed significantly from the age at monotonic testing (1 year) to the age at cyclic loading (2 years).

The dimensions of the fatigue specimens were kept the same as those used in [19]. Using the same concrete and the same specimen shape made it possible to use the known strength values in calculating the loads to be applied. However, while four different sizes were tested in [19], here only three different sizes $D$ could be tested, viz., 40, 93 and $215 \mathrm{~mm}$. The thickness $b$ of all beams was $1.58 \mathrm{in.}(40 \mathrm{~mm})$ and the length-to-depth ratio $s d$ was 2.4. For each beam, a notch of depth $\alpha_{0}=a_{0} / D=0.3$ was also pre-cut with the saw. The material properties as obtained in [19] are summarized in Table 1.

\begin{tabular}{ll}
\hline Young's modulus $[\mathrm{MPa}]$ & 41240 \\
Poisson's ratio & 0.18 \\
Density $\left[\mathrm{kg} / \mathrm{m}^{3}\right]$ & 2400 \\
Compressive strength $f_{c}^{\prime}[\mathrm{MPa}]$ & 55.6 \\
SEL parameter $B f_{t}^{\prime}[\mathrm{MPa}]$ & 3.98 \\
Transitional size for monotonic fracture $D_{0 m}[\mathrm{~mm}]$ & 143.8 \\
\hline
\end{tabular}

Table 1: Material properties of concrete from [19]

To minimize the influence of scatter, 6 specimen of each size were tested for fatigue. This is an improvement over previous data sets for concrete, in which only 1 or 2 specimen were tested per size. Fatigue testing was conducted under load control in an MTS closed-loop testing machine, by using a load cell of capacity of 5 kips $(20 \mathrm{kN})$. For each size, the applied cyclic loading had an upper limit of $85 \%$ and lower limit of $5 \%$ of the mean peak load for that size (as obtained from [19]). Thus, the ratio of $\Delta \sigma$ to the mean $\sigma_{N}$ was kept constant for all the beam sizes. This was done in order to load the cyclic FPZ's in each specimen at roughly the same frequency. The lower limit had to be kept finite rather than zero in order to avoid separation between the specimen and the loading fixture.

The frequency of loading was $0.1 \mathrm{~Hz}$ and a linear ramp load was used. An MTS extensometer was attached to the bottom surface of the beam, to measure the crack mouth opening displacement (CMOD). The CMOD values would then yield the effective crack length, via the compliance calibration method. In all the tests, the stroke (displacement of the piston), the CMOD, time and force were recorded at every peak and valley of the load history. Fig. 1 shows a photograph of a medium size beam being tested. 


\subsection{Test results: fatigue lifetimes}

The fatigue tests were run until complete failure of the specimens. After failure, the cracking pattern was analyzed for each specimen. Fig. 2 shows one failed specimen of each size. It is seen that the crack path was tortuous but clearly followed the ligament center-line for each size. All the other specimen also failed in a similar fashion. Thus the geometrical similarity of the failure is verified. We first present the recorded lifetimes for all the tested specimen in Table 2.

\begin{tabular}{cccc}
\hline Specimen & Small & Medium & Large \\
\hline 1 & 102 & 685 & 1451 \\
2 & 21 & 394 & 20 \\
3 & 1622 & 631 & 33 \\
4 & 815 & 1452 & 465 \\
5 & 2084 & 155 & 37 \\
6 & 1354 & 64 & 504 \\
Mean $\bar{N}_{f}$ & 1000 & 564 & 418 \\
Standard deviation & 835 & 501 & 553 \\
COV [\%] & 83.53 & 88.87 & 132.17 \\
\hline
\end{tabular}

Table 2: Experimental values of fatigue lifetimes for concrete beams of various sizes

It can be seen that although the fracture pattern is very consistent, the scatter in lifetimes is very high, spanning more than two orders of magnitude, for each size. The $\mathrm{CoV}$ of the fatigue lifetimes is more than $100 \%$ (although the $\mathrm{CoV}$ of logarithm of lifetime is $\approx 20 t o 30 \%$ ). The average lifetime $\overline{N_{f}}$, is seen to decrease with increasing size. This agrees with the predictions of the microplane model in [16].

\subsection{Test results: structural compliance evolution}

During each test, the CMOD at maximum load was continuously monitored to track the accumulating damage. In most specimens, the CMOD was seen to increase rapidly in the first few cycles, followed by a considerable slow down. After this, the growth per cycle was slow and regular for many cycles (this behavior is indicative of a crack growth rate following a power law,i.e., Paris law). As the beam approached its lifetime, the CMOD, and also its growth rate per cycle, was seen to increase rapidly until catastrophic failure.

The CMOD in each cycle was then used in the compliance calibration method to estimate the instantaneous effective crack length. This is necessary since it is virtually impossible to directly measure the crack length for concrete, due to the diffuse nature of fracture. The compliance calibration curve for each size was generated numerically using the known elastic stiffness. For various values of the notch length $a_{0}$, the CMOD compliance $C_{C M O D}$, equal to the slope of the load vs. CMOD plot, was determined by simulating one loading cycle at a very low maximum load. Then, a cubic polynomial relating $C_{C M O D}$ to $a_{0}$ was obtained by regression. This process was repeated for each beam size. Fig. 3 shows the numerically generated compliance calibration curves for each size.

To determine the instantaneous effective crack length $a$ during any loading cycle of the fatigue test, first the $C_{C M O D}$ was calculated in that cycle as the inverse slope of the unloading segment of 
the measured load-CMOD curve. When substituted into the previously fitted calibration polynomial, it provided the effective crack length in that cycle. Figs. 4a-c show the evolution of normalized crack length for each specimen of small, medium and large size respectively. The trend is fairly consistent in all cases. The rate of crack growth is very slow initially but accelerates towards the end, agreeing with previous observations $[11 ; 12 ; 13]$.

\subsection{Test results: Paris law and size effect}

To plot Paris law, the effective crack length was calculated from the crack growth rate $\Delta a / \Delta N$ using $\Delta N=10$. The corresponding amplitude of the stress intensity factor was determined as

$$
\Delta K=\Delta \sigma \sqrt{D} k(\alpha)
$$

where $k(\alpha)$ is the dimensionless stress intensity factor for this geometry. It has the form;

$$
k(\alpha)=\frac{\sqrt{\alpha} p_{s d}}{(1+2 \alpha)(1-\alpha)^{3 / 2}}
$$

where $p_{s d}$ depends on the span-to-depth ratio $s d$ [1], which is here 2.4. Approximately, though with high accuracy, it may be calculated as

$$
\begin{aligned}
& p_{s d}=p_{\infty}+\frac{4}{s d}\left(p_{4}-p_{\infty}\right) \\
\text { where } & p_{4}=1.9-\alpha\left[-0.089+0.603(1-\alpha)-0.441(1-\alpha)^{2}+1.223(1-\alpha)^{3}\right] \\
\text { and } & p_{\infty}=1.989-\alpha(1-\alpha)\left[0.448-0.458(1-\alpha)+1.226(1-\alpha)^{2}\right]
\end{aligned}
$$

Figs. 5a-c show the Paris law plots obtained for individual specimens of small, medium and large sizes. Unfortunately, for specimens with lifetimes smaller than 100 cycles, the test data are insufficient to generate a meaningful Paris law plot, since the Paris regime consists of even fewer cycles. Therefore, these data had to be excluded from the Paris law analysis. Notwithstanding, they are still included in the lifetime analysis.

The Paris law plots for each size are seen to be very scattered, especially in the intercept, $\ln C$. The individual values of $C, \ln C$ and $m$ for small, medium and large sizes are shown in Tables 3 , 4 and 5 respectively. The average values $\bar{C}, \ln \bar{C}$ and $\bar{m}$ are also shown, along with the standard deviation. Similar to the lifetimes, the standard deviation in $C$ is seen to be very high. Interestingly, the standard deviation of the exponent is much lower. This indicates that the scatter in lifetimes is directly related to the scatter in the Paris law intercept, $\ln C$, and not the exponent $m$.

\subsection{Test results: transitional size $D_{0 c}$ and $R_{f}$}

In the previous studies, the values of $D_{0 c}$ and $R_{f}$ were obtained by normalizing Paris law plots of individual specimens, not accounting for the scatter. As an improvement, here we use the average values of $\ln \bar{C}$ and $\bar{m}$ to determine $D_{0 c}$ and $R_{f}$. Accordingly, for each size, one may plot a straight line that has a slope equal to $\bar{m}$ and an intercept equal to $\ln \bar{C}$; see Fig. 6a. Then, these average Paris law plots are normalized using Eq. 7, to obtain the value of $D_{0 c}$ iteratively. For this data set, $D_{0 c}$ turns out to be $17.16 \mathrm{~mm}$. The normalized plots are shown in Fig. 6b (Fig. 6c will be explained later). Referring to table 1 , the ratio $R_{f}=D_{0 c} / D_{0 m}$ is equal to $\mathbf{0 . 1 2}$. This implies that the cyclic FPZ is $\approx$ 8-times smaller than the monotonic one.

This finding contrasts with the previous one in [12]; in which $R_{f}$ was $\approx 0.9$, while in [13] it was $\approx 1.5$. One possible cause of this contrast is that here the mean behavior of several specimens 


\begin{tabular}{cccc}
\hline Specimen & $C$ & $\ln C$ & $m$ \\
\hline & & & \\
1 & $\mathrm{NA}$ & $\mathrm{NA}$ & $\mathrm{NA}$ \\
2 & $\mathrm{NA}$ & $\mathrm{NA}$ & $\mathrm{NA}$ \\
3 & -29.77 & $1.18 \mathrm{E}-13$ & 8.33 \\
4 & -29.81 & $1.13 \mathrm{E}-13$ & 8.65 \\
5 & -31.87 & $1.44 \mathrm{E}-14$ & 9.4 \\
6 & -30.26 & $7.39 \mathrm{E}-14$ & 7.99 \\
Mean & -30.42 & $7.98 \mathrm{E}-14$ & 8.59 \\
Standard deviation & & $4.78 \mathrm{E}-14$ & 0.6 \\
COV [\%] & & 59.93 & 7.01 \\
\hline
\end{tabular}

Table 3: Experimental values of Paris law parameters for concrete beams of small size

\begin{tabular}{cccc}
\hline Specimen & $C$ & $\ln C$ & $m$ \\
\hline 1 & -32.22 & $1.016 \mathrm{E}-14$ & 8.54 \\
2 & -29.38 & $1.74 \mathrm{E}-13$ & 7.75 \\
3 & -31.59 & $1.91 \mathrm{E}-14$ & 8.68 \\
4 & -29.83 & $1.11 \mathrm{E}-13$ & 7.92 \\
5 & -32.52 & $7.53 \mathrm{E}-15$ & 8.9 \\
6 & $\mathrm{NA}$ & $\mathrm{NA}$ & $\mathrm{NA}$ \\
Mean $\bar{N}_{f}$ & -31.11 & $6.43 \mathrm{E}-14$ & 8.36 \\
Standard deviation & & $7.48 \mathrm{E}-14$ & 0.49 \\
COV [\%] & & 116.32 & 5.96 \\
\hline
\end{tabular}

Table 4: Experimental values of Paris law parameters for concrete beams of medium size

for each size is considered (it may be even better to test next time 20 or even 100 specimens). Anyway, the present $R_{f}$ values appear to be more reliable than the previous values. We now seek a numerical verification, using the recently developed model for quasibrittle fatigue in [16].

\section{Predictions using the microplane fatigue damage model M7}

\subsection{Model overview}

The microplane model M7 [20; 21] is the latest version in a series of progressively improved microplane models labeled M0, M1, M2...M6, developed primarily for concrete [22]. The fundamental idea of the microplane model is to express the constitutive law in terms of the vectors of stress and strain, instead of tensors. These vectors act on a generic plane of any orientation in the material microstructure, called the "microplanes". Considering all such planes captures the interactions between various orientations, which makes the model semi-multiscale in nature (a fully multi-scale model would have to capture also the interactions at distance). The microplane model, supplemented by a suitable localization limiter with a material characteristic length, has been proven to give realistic predictions of the constitutive and damage behavior of quasi-brittle materials over a 


\begin{tabular}{cccc}
\hline Specimen & $C$ & $\ln C$ & $m$ \\
\hline & & & \\
2 & -32.09 & $1.15 \mathrm{E}-14$ & 8.03 \\
3 & $\mathrm{NA}$ & $\mathrm{NA}$ & $\mathrm{NA}$ \\
4 & $\mathrm{NA}$ & $\mathrm{NA}$ & $\mathrm{NA}$ \\
5 & -31.16 & $2.93 \mathrm{E}-14$ & 7.99 \\
6 & $\mathrm{NA}$ & $\mathrm{NA}$ & $\mathrm{NA}$ \\
Mean & -31.8 & $1.55 \mathrm{E}-14$ & 8.03 \\
Standard deviation & -31.68 & $1.88 \mathrm{E}-14$ & 8.02 \\
COV [\%] & & $1.26 \mathrm{E}-14$ & 0.028 \\
\end{tabular}

Table 5: Experimental values of Paris law parameters for concrete beams of large size

broad range of monotonic, multi-axial loading scenarios [21]. It was recently enhanced to also yield accurate prediction of the fatigue response of statistically isotropic quasibrittle materials such as concrete [16]. In the formulation for fatigue, the damage law is defined on the microplane level as:

$$
\frac{E_{N}}{E_{N 0}}=\exp \left(-A \epsilon_{N}^{0+}\right)\left(\frac{1}{1+r \zeta^{q}+r^{2} \zeta^{2 q}}\right)
$$

Here $E_{N}$ is the damaged normal microplane modulus, $E_{N 0}$ is the original undamaged modulus; $\epsilon_{N}^{0+}$ is the maximum strain that can characterize tensile damage on that microplane; $A$ is a material constant (which is referred to as $c_{13}$ in M7 [20]); and $\zeta$ is the cyclic damage measure, calculated as the path length of the inelastic volumetric strain, in strain space. Thus, if $\zeta_{0}$ is the value of the damage parameter at the beginning of a time increment, then the value $\zeta$ at the end is given by $\zeta=\zeta_{0}+\mathrm{d} \zeta$, where $\mathrm{d} \zeta=\left|\mathrm{d} \epsilon_{V}^{\prime \prime}\right|$ is the increment of the inelastic volumetric strain. Here, $r$ and $q$ are the two new material parameters, introduced for fatigue. It is found that $r$ affects mainly the predicted lifetime, whereas $q$ mainly Paris law exponent $m$.

The present model gave realistic predictions of fatigue crack growth in normal and high strength concretes up to several thousand load cycles, including material hysteresis, structure compliance evolution and Paris law with a high exponent, which is typical of quasibrittle materials. It was also shown to apply to sudden overloads, and to predict well the residual strength degradation of structures. The size effect in Paris law predicted by the model also agreed qualitatively with the experiments. These capabilities will now be evaluated quantitatively.

\subsection{Predicting $R_{f}$ using transitional sizes $D_{0 c}$ and $D_{0 m}$}

We try to predict $R_{f}$, as $D_{0 c} / D_{0 m}$, where $D_{0 m}$ is obtained by fitting the data on the size effect on monotonic strength, and $D_{0 c}$ by size normalization of the Paris law plots. First, the free parameters of the microplane model [21] are calibrated so as to match the monotonic loaddisplacement response of the three beams reported in [19]. The radial scaling parameter $k_{1}$ of microplane model M7, defined in [21], had to be adjusted in order to predict the monotonic peak loads of the beams within acceptable error; its value came to be $k_{1}=105 \times 10^{-6}$. All the other parameters of model M7 were equal to their default values listed in [21].

Both the monotonic and fatigue crack growth in the beams was simulated by the crack band model [23], which is the simplest and, in engineering practice, the most widely used model to 
introduce a localization limiter for softening damage. Comparison of the simulations with the average of experimental results, seen in Fig. 7 and Table 1, shows very good agreement. From the predicted peak loads, the size effect law parameters are obtained by linear regression in the plot of $1 / \sigma_{N}^{2}$ vs. $D$. This yields $B f t^{\prime}=1 / \sqrt{A_{1}}=4.05 \mathrm{MPa}$ and $D_{0}^{m}=A_{1} / B_{1}=123.06 \mathrm{~mm}$ where $A_{1}$ and $B_{1}$ are the intercept and slope of the linear fit [1]).

Next, as described in [16], fatigue simulations were performed for the beams of three sizes using the calibrated model. The applied cyclic loading had maximum and minimum load limits corresponding to $85 \%$ and $5 \%$ of the peak load, as in the tests. The load frequency was $5 \mathrm{~Hz}$, which ensured absence of inertia effects. From the tests, the average lifetime of the small beam was 1000 cycles.

The damage law parameters were adjusted until the predicted lifetime and Paris law exponent became close enough. For $r=1000$ and $q=2.5$, the model predicted failure in 889 cycles for the small size, which is a reasonable agreement. The lifetimes predicted for the medium and large size beams were 855 and 730 . These lifetimes are higher than the test averages (564 and 418), but the difference is not surprising in view of the huge scatter in lifetimes. What matters for lifetimes is the order of magnitude, and this is predicted correctly. Also, both the predicted and observed experimental lifetimes decrease with an increase in size.

For each size, the normalized effective crack length $\alpha$ was determined by means of the compliance calibration method, after which the Paris law was plotted, as explained earlier. Fig. 8a shows the predicted Paris law plots, in its unadjusted form. The predicted Paris law exponents for the three sizes are 7.11, 7.26 and 7.54 , and the values of intercept $\ln C$ are $-27.63,-28.45$ and -29.73 . These agree reasonably well with the test data from Tables 3, 4 and 5. This reaffirms that the model predicts satisfactorily the size effect in Paris law coefficient $C$. Also, no size effect is predicted to occur in exponent $m$, which is consistent with experimental observations.

The plots normalized according to Eq. 7 are shown in Fig. 8b (Fig. 8c will be explained later). This yields $D_{0 c}=9.23 \mathrm{~mm}$, i.e. $R_{f}=\mathbf{0 . 0 8}$. The agreement with the test value of $\mathbf{0 . 1 2}$ is close, further validating the model. Since the model predictions have minimal scatter, this also shows that the value of $R_{f}$ from the tests is much more reliable. This emphasizes that it is absolutely necessary to use the average response from many tests to determine $D_{0 c}$ and $R_{f}$ reliably and prevent misleading inferences on the cyclic FPZ size.

\subsubsection{Predicting $R_{f}$ from FPZ sizes}

While the previous section showed good agreement between the tests and the model, the question that now arises is whether the cyclic FPZ is really 8 to 12 times smaller than the monotonic FPZ. We now check this by identifying the FPZ sizes from the modeling results. It should be pointed out here, that in [13], a laudable attempt was made to experimentally measure the FPZ sizes using the digital image correlation (DIC) technique. But the accuracy of these measurements could not be guaranteed given the extremely diffuse and blunt nature of the fracture process. Besides, the start and end points of the FPZ were obtained from displacement profiles in the ligament.

Correctly, the FPZ size must be estimated from the stress profiles, which can be obtained only from numerical simulations. Accordingly, the FPZ size is here determined from the predicted profile of stress $\sigma_{x}$ at peak load across the ligament. The monotonic FPZ size, $L_{f m}$, at the peak load state is equal to the distance between the stress peak and the notch tip, as shown in Fig. 9a. 
c1 For the small, medium and large sizes, $L_{f m}$ was obtained as 8,15 and $32 \mathrm{~mm}$. The change from small to large size may seem surprisingly large, but not if one notes that, from the monotonic size effect fitting, $c_{f m}$ was estimated as $23.95 \mathrm{~mm}$. According to [24; 25], the fully developed FPZ size should be $\approx 2$ to 2.5 times $c_{f m}$ i.e. about 48 to $60 \mathrm{~mm}$. This means that the FPZ at peak load of small quasibrittle specimens of positive geometry is far from completely developed. This is plausible because, in a small specimen, there is not enough 'room' for the FPZ to develop [13]. This observation is supported by simulations showing that, at peak load of small specimens, the cohesive normal stress at the notch tip, $\sigma_{n o t c h}$, is not yet zero and in fact quite large.

To measure the cyclic FPZ size, the predicted stress profiles are plotted at various stages throughout the fatigue simulation, as shown in Fig. 9b for the largest specimen and for cycles number 240, 720 and 889 (the terminal cycle). For any given cycle, the size of the cyclic FPZ is computed as the distance between points A and B, such that point A corresponds to the peak of the $\sigma_{x}$ profile, and point B corresponds $\sigma_{x}=\sigma_{n o t c h}$ for that size, in that cycle. It was seen that the cyclic FPZ size was not constant throughout the simulated lifetimes, but grew towards the end, approaching the monotonic FPZ size. Therefore, the average cyclic FPZ size was calculated for all three sizes in every $40^{\text {th }}$ cycles. The average cyclic FPZ sizes $L_{f c}$ for small, medium and large sizes were $4.375,8.75$ and $18.65 \mathrm{~mm}$, respectively, and the FPZ size ratios $R_{f}=L_{f}^{c} / L_{f}^{m}$ are $\approx 0.55,0.58$ and 0.58. So, on the average, the cyclic FPZ is about $\mathbf{0 . 5 7}$ times the monotonic FPZ.

The difference of this FPZ size ratio from ratio $R_{f}$ obtained as the ratio of transitional sizes (which is 0.12 from experiments and 0.08 from modeling) is rather big and puzzling. It implies that the $D_{0 c}$ obtained from the size adjusted Paris law is not proportional to the cyclic FPZ size. So, it seems that the discrepancy might be due to the very method used for obtaining $D_{0 c}$. We check it next.

\section{Proposed correction to the size adjusted Paris law}

The dimensional analysis presented in [13] is now revisited to explain the observed discrepancy in the values of $R_{f}$. Bažant et al. [26] justified theoretically first the Charles-Evans law for static crack growth and subsequently, by an analogous argument, the Paris law for cyclic crack growth. They started from the nano-scale, based on equating the energy dissipation of all $n_{a}$ active nanoscale cracks in the cyclic FPZ the macro-scale energy dissipation per load cycle (see also [27; 13]). This energy is equal to $U_{c, \infty} \mathrm{d} a / \mathrm{d} N$ for a purely brittle material following LEFM, and to $U_{c} \mathrm{~d} a / \mathrm{d} N$ for a quasibrittle material, $U_{c}$ being the size dependent fatigue fracture energy.

The energy dissipation for the $i^{t h}$ nano-crack, for one load cycle is given by $U_{a} \mathrm{~d} a_{i} / \mathrm{d} N$ where $U_{a}$ is the critical energy dissipation associated with one nano-crack, and $\mathrm{d} a_{i} / \mathrm{d} N$ is the extension of the $i^{t h}$ nano-crack per cycle. The main point is that the frequency of the breaking of atomic bonds can be derived from Kramer's rule. This leads the Paris law (as well as the Charles-Evans), though with the exponent of $2[26 ; 27]$ for the nano-scale. So, the energy dissipated per cycle for a nano-crack is proportional to $\Delta K_{a}^{2}$. Considering that the atomic scale $\Delta K_{a}$ is roughly proportional to the macro scale $\Delta K$, one gets the atomic scale Paris law:

$$
\frac{\mathrm{d} a}{\mathrm{~d} N}=n_{a} \frac{A \Delta K^{2}}{U_{c}}
$$

\footnotetext{
${ }^{\text {c1 }}$ Fig. 9 is updated
} 
where $A$ is a material constant. The increase of exponent from the atomic scale to the RVE scale is then explained, for Paris law [27] (as well as Charles-Evans law), upon noting that all the atomic scale cracks within the FPZ must dissipate, within each cycle (or within a unit time), the same energy per cycle as the macro-FPZ itself [26;27].

In a previous study of Paris law size effect [13], the functional form of Paris law was derived by considering $n_{a}$ as the dependent variable and $\Delta K$ as one of the dependent variables. While this choice is plausible for the LEFM case, the dependence of $n_{a}$ for a quasibrittle material must be normalized with respect to the structure size by considering $\Delta K / K_{I C}$ rather than $\Delta K$. So, $\Delta K_{1}=\Delta K / K_{I C}$ needs to be introduced and the dimensional analysis is now revisited using $\Delta K_{1}$ instead of $\Delta K$.

Accordingly, with $n_{a}$ as the dependent variable, the following independent variables are now considered: 1) $\Delta K_{1}$;2) the fatigue fracture energy, $U_{c}$ (which is size dependent for a quasibrittle material); 3) the critical stress intensity factor for a specimen of infinite size, $K_{I F}$; 4) Young's modulus $E$; and 5) load ratio $R$. So,

$$
n_{a}=f\left(\Delta K_{1}, U_{c}, K_{I F}, E, R\right)=f\left(\Delta K_{1}, U_{c}, K_{I F}, E\right) \text { for a fixed R ratio }
$$

Standard dimensional analysis according to [10] and [13] gives,

$$
n_{a}=\Phi\left(\Delta K_{1}, \frac{U_{c} E}{K_{I F}^{2}}\right)=\Delta K_{1}^{p}\left(\frac{U_{c} E}{K_{I F}^{2}}\right)^{q} \quad \text { Assuming self similarity of the function } \Phi
$$

Then, replacing $\Delta K$ by $\Delta K_{1}$ and substituting (16) into (14), one gets

$$
\frac{\mathrm{d} a}{\mathrm{~d} N}=\frac{A \Delta K_{1}^{2}}{U_{c}} \Delta K_{1}^{p}\left(\frac{U_{c} E}{K_{I F}^{2}}\right)^{q}=C \Delta K_{1}^{p_{1}} U_{c}^{q_{1}}
$$

where is $C$ is a new constant obtained by grouping all the other constants $\left(q_{1}=q-1, p_{1}=p+2\right)$. Substitution of the expressions for $U_{c}$ and $\Delta K_{1}$ furnishes,

$$
\frac{\mathrm{d} a}{\mathrm{~d} N}=C_{1} \Delta K^{p_{1}}\left(\frac{D+D_{0}^{m}}{D}\right)^{p_{1} / 2}\left(\frac{D}{D+D_{0 c}}\right)^{q_{1}}
$$

where $C_{1}=C\left(U_{c, \infty}\right)^{q_{1}}$. As the simplest case, consider that $p_{1} / 2=q_{1}$. This yields,

$$
\frac{\mathrm{d} a}{\mathrm{~d} N}=C_{1}(\Delta K)^{m}\left(\frac{D+D_{0 m}}{D+D_{0 c}}\right)^{m / 2}
$$

This is the new size-adjusted Paris law (exponent $m$ is a material constant). This law involves both $D_{0 m}$ and $D_{0 c}$. The expression for the size adjusted amplitude of stress intensity factor is,

$$
\Delta K_{D}=\Delta K\left(\frac{D+D_{0 m}}{D+D_{0 c}}\right)^{1 / 2}
$$

Note that this reduces to $\Delta K_{D}=\Delta K$ for LEFM since $D_{0 m}=D_{0 c}=0$. This check is important since for LEFM there cannot be any size dependence in $\mathrm{d} a / \mathrm{d} N$. 


\section{1. $R_{f}$ based on modified size adjusted Paris law}

We now obtain $D_{0 c}$ and $R_{f}$ using equation 19 from both experiments and simulations. The revised normalized plot from experiments is shown in Fig. 5c. Here, the value of $D_{0 m}$ is an input, since it is known from the monotonic size effect fitting. $D_{0 c}$ is determined as before, iteratively, until the three plots merge into one. This occurs at $D_{0 c}=67 \mathrm{~mm}$ (for $D_{0 m}=143 \mathrm{~mm}$ ). Thus the new ratio $R_{f}$ is equal to $\mathbf{0 . 4 7}$. Fig. 8c shows the modified size normalization of the Paris law, obtained from simulations. For the model, using $D_{0 m}=123 \mathrm{~mm}$, the new values of $D_{0 c}$ and $R_{f}$ are $75.06 \mathrm{~mm}$ and $\mathbf{0 . 6 1}$. The new values of $R_{f}$ now agree very well with the $R_{f}$ obtained by measuring the FPZ sizes, which was $\mathbf{0 . 5 7}$ (see also [28] for a similar numerical verification with HSC beams, which confirms that the modified form yields $D_{0 c}$ values proportional to the cyclic FPZ size). This is summarized in the following table 6 : ${ }^{\mathrm{c} 1}$

\begin{tabular}{|l|l|l|l|}
\hline & $\begin{array}{l}R_{f} \text { by normaliz- } \\
\text { ing Paris law (ex- } \\
\text { periments) }\end{array}$ & $\begin{array}{l}R_{f} \text { by normal- } \\
\text { izing Paris law } \\
\text { (modeling) }\end{array}$ & $\begin{array}{l}R_{f} \text { by measur- } \\
\text { ing FPZ sizes } \\
\text { (modeling) }\end{array}$ \\
\hline By using eq. (7) & 0.12 & 0.08 & 0.57 \\
By using eq. (19) & 0.47 & 0.61 & 0.57 \\
\hline
\end{tabular}

Table 6: Summary of $R_{f}$ values from experiments and modeling by using equations 7 and 19

This agreement may be considered as a good enough verification of the modified form, given the huge scatter in the test data. Also, the FPZ measurement from the modeling has some discretization error (equal to about half the mesh size). This could be reduced by a more refined mesh, but is nevertheless much smaller compared to the difference between the original and newly calculated $R_{f}$ values (which differ by over $500 \%$ ).

It may be argued that, ideally, the fully developed FPZ from larger sizes should be used to find $R_{f}$. But here it is seen that the ratio $R_{f}$ stays unchanged for all the three sizes. Therefore, one may assume that it should not be too different even for fully developed FPZs. Notwithstanding this, it may still be concluded that the modified form of the size adjusted Paris law is a possible explanation of why $D_{0 c}$ from the original form is not proportional to the cyclic FPZ size.

It should be possible to verify the present modified form on other quasibrittle materials, provided the experimental scatter is accounted for. Of course, several specimens for each of several sufficiently different sizes would have to be tested, upon which only the average Paris law plots would be normalized.

\section{Size effect in fatigue lifetime}

As another improvement over previous studies, let us now analyze the size effect in fatigue lifetime, as predicted by the size-adjusted Paris law. Consideration is limited to the case in which the applied loads are scaled strictly according to the specimen strength, i.e., $\Delta \sigma / \sigma_{N}$ is kept constant (while $\Delta \sigma$ is not constant). So, let $\Delta \lambda$ be $=\Delta \sigma / \sigma_{N}=$ constant.

\footnotetext{
${ }^{\mathrm{c} 1}$ Added new table (Table 6)
} 
Let us begin with the LEFM case, in which there is no size effect in Paris law. The separation variables in Eq. (1) gives:

$$
\frac{D \mathrm{~d} \alpha}{C \Delta K^{m}}=\mathrm{d} N
$$

where $\alpha$ is the normalized crack length $=a / D$. Now, since $\Delta K=\Delta \sigma \sqrt{D} k(\alpha)$ and $\Delta \sigma=\Delta \lambda \sigma_{N}$,

$$
\frac{\mathrm{d} \alpha}{C\left(\Delta \lambda \sigma_{N} \sqrt{D} k(\alpha)\right)^{m}}=\frac{\mathrm{d} N}{D}
$$

To obtain the lifetime, $N_{f}$, we integrate the left-hand side from the initial to the final relative crack length, i.e., from $\alpha_{0}$ to $\alpha_{c}$, and the right-hand side from 0 to $N_{f}$. So we have,

$$
\frac{1}{C_{1}} \int_{\alpha_{0}}^{\alpha_{c}} \frac{\mathrm{d} \alpha}{k(\alpha)^{m}}=\frac{1}{D} \int_{0}^{N_{f}} \mathrm{~d} N=\frac{N_{f}}{D}
$$

where $C_{1}=C\left(\Delta \lambda \sigma_{N} \sqrt{D}\right)^{m}$. This is constant since, for LEFM, $\sigma_{N} \propto 1 / \sqrt{D}$. Also, for LEFM, the final critical crack length $a_{c}$ scales linearly as $D$ making $\alpha_{c}$ a constant. Thus the entire left-hand side is a constant, $C_{2}=1 / C_{1} \int_{\alpha_{0}}^{\alpha_{c}} \frac{\mathrm{d} \alpha}{k(\alpha)^{m}}$. This means that, for LEFM, $N_{f} \propto D$, or the plot of $N_{f} / C_{2}$ vs. $D$ is a straight line. If the size doubles, with appropriately scaled loading, the lifetime will also double.

Let us now extend the foregoing to the quasibrittle case, which exhibits a size effect in Paris law. First analyze the original form, i.e. Eq. (7). Similar to the LEFM case, the separation of variables gives,

$$
\frac{D \mathrm{~d} \alpha}{C \Delta K^{m}}=\mathrm{d} N\left(1+\frac{D_{0 c}}{D}\right)^{m / 2}
$$

Now, using $\Delta K=\Delta \sigma \sqrt{D} k(\alpha) ; \Delta \sigma=\Delta \lambda \sigma_{N}$, accounting for the size effect in $\sigma_{N}$, and integrating throughout the crack growth history, one obtains

$$
\frac{1}{C_{1 a}} \int_{\alpha_{0}}^{\alpha_{c}} \frac{\mathrm{d} \alpha}{k(\alpha)^{m}}=\frac{1}{D}\left(\frac{D+D_{0 c}}{D+D_{0 m}}\right)^{m / 2} N_{f}
$$

Here $C_{1 a}$ is a constant equal to $C\left(\Delta \lambda B f_{t}^{\prime} \sqrt{D_{0 m}}\right)^{m}$. Unlike the LEFM case, here the integral on the left-hand side cannot be considered constant. This is because $\alpha_{c}$ also exhibits a size effect! Thus an analytical form is impossible. Denoting the integral on the left-hand side as $F\left(\alpha_{c}\right)$ and realizing that $\alpha_{c}$ depends on $D$, we may write, for the fatigue lifetime,

$$
N_{f}=\frac{1}{C_{1 a}} D\left(\frac{D+D_{0 m}}{D+D_{0 c}}\right)^{m / 2} F\left(\alpha_{c}\right)
$$

This expression gives the size effect in fatigue lifetime predicted by the existing form of the sizeadjusted Paris law.

Next analyze the same for the modified form, i.e., for Eq. (19. Repeating the separation of variables yields

$$
\frac{D \mathrm{~d} \alpha}{C \Delta K^{m}}=\left(\frac{D+D_{0 m}}{D+D_{0 c}}\right)^{m / 2} \mathrm{~d} N
$$

Then, simplifying as before, and integrating the crack growth history, one gets

$$
\frac{1}{C_{1 a}} \int_{\alpha_{0}}^{\alpha_{c}} \frac{\mathrm{d} \alpha}{k(\alpha)^{m}}=\frac{1}{D^{(1-m / 2)}}\left(\frac{1}{D+D_{0 c}}\right)^{m / 2} N_{f}
$$


So, for fatigue lifetime,

$$
N_{f}=\frac{1}{C_{1 a}} D^{(1-m / 2)}\left(D+D_{0 c}\right)^{m / 2} F\left(\alpha_{c}\right)
$$

It is interesting to note that mere consideration of $D_{0 c}$ in the size-adjusted Paris law leads to a dependence on both $D_{0 c}$ and $D_{0 m}$ for the lifetime. On the other hand, considering in the Paris law both $D_{0 c}$ and $D_{0 m}$ yields $D_{0 c}$ as the only variable affecting the lifetime.

\subsection{Numerical estimates}

Although the exact analytical solutions cannot be obtained, one can examine the foregoing formulation numerically. The main challenge lies in estimating $F\left(\alpha_{c}\right)$. To this end, assume that, in the final cycle, when the effective crack length equals $a_{c}$, the applied maximum load $\sigma_{\text {max }}$ is equal

to the monotonic strength of the beam. So, in the terminal cycle, $K_{I C}=\sigma_{\max } \sqrt{D} k\left(\alpha_{c}\right)$ where

$$
\alpha_{c}=k^{-1}\left(\frac{K_{I C}}{\sqrt{D} \sigma_{\max }}\right)
$$

To estimate $\alpha_{c}$ for various sizes, the data for the concrete analyzed here are used (see the material properties in Table 7 ).

\begin{tabular}{ll}
\hline Initial normalized crack length $\alpha_{0}$ & 0.3 \\
Applied max load as fraction of strength $\sigma_{\max } / \sigma_{N}$ & 0.85 \\
Paris law exponent $m$ & 7.2 \\
Cyclic transitional size for original theory $D_{0 c}[\mathrm{~mm}]$ & 9.23 \\
Cyclic transitional size for modified theory $D_{0 c}[\mathrm{~mm}]$ & 75 \\
Monotonic transitional size $D_{0 m}[\mathrm{~mm}]$ & 123 \\
SEL parameter $B f_{t}^{\prime}[\mathrm{MPa}]$ & 4.05 \\
Fracture toughness $K_{I C}[\mathrm{MPa} \sqrt{\mathrm{m}}]$ & 43.57 \\
Initial dimensionless SIF $k_{0}$ & 0.9695 \\
\hline
\end{tabular}

Table 7: Material parameters of concrete under consideration, used for calculating the size effect in fatigue lifetime

Then, substitution of the value of $K_{I C}$ from Table 7 into Eq. (30) provides the value of $\alpha_{c}$ for different sizes. The size effect in $\alpha_{c}$ is shown in Fig. 10a. Note that, for larger sizes, the size effect vanishes and $\alpha_{c}$ becomes constant, consistent with LEFM. Then these values of $\alpha_{c}$ are used to determine $F\left(\alpha_{c}\right)$ by means of numerical integration (with Simpson's rule), and thus to estimate $N_{f} / N_{0}$ where $N_{0}=1 / C_{1 a}$. These calculations have been performed using both the original and modified forms of the Paris law.

The results are found to be rather interesting, as shown in Fig. 10b. Both forms are seen to predict a non-monotonic size effect in lifetime. For smaller sizes, both forms predict a decrease in lifetime with the structure size. This is consistent with both the modeling results and the average values from experiments. But for larger sizes, the lifetime reaches a minimum, and then starts to increase, eventually becoming linear, consistent with LEFM (shown by a dashed line in Fig. 10b).

Note that this is the size effect in lifetime that occurs when $\Delta \sigma / \sigma_{N}$ remains constant. This should not be confused with the size effect in lifetime where $\Delta \sigma$ is held constant, for which the lifetime obviously decreases with increasing structure size, and does so monotonically [27].

No experimental data sets seem to exist to verify this non-monotonic size effect. Verification by modeling may be possible. But simulating fatigue in specimens of very large sizes is computationally very demanding. 


\section{The question of size effect in fatigue threshold}

The previous as well as the modified form inevitably has a limitation that becomes evident in analyzing the size effect on fatigue threshold [27]. The fatigue threshold $\Delta K_{t h}$ is defined as the largest amplitude $\Delta K$ at which no fatigue crack growth can be discerned. To obtain the size effect in $\Delta K_{t h}$, let us write

$$
\frac{\mathrm{d} a}{\mathrm{~d} N}=\nu_{t h}=C\left(\Delta K_{t h}\right)^{m} \phi(D)
$$

where $\phi(D)$ is a function of structure size, different for the two size-adjusted Paris laws, and $\nu_{t h}$ is the threshold rate of fatigue crack growth. For the original form,

$$
\nu_{t h}=C\left(\Delta K_{t h}\right)^{m}\left(1+\frac{D_{0 c}}{D}\right)^{m / 2} \quad \text { or } \quad \frac{\Delta K_{t h}}{\Delta K_{0}}=\frac{1}{\sqrt{1+D_{0 c} / D}}
$$

where $\Delta K_{0}=\left(\nu_{t h} / C\right)^{1 / m}$. This happens to agree with the data reported in [29] on the effect of crack length on fatigue threshold for very short cracks in metals (since the cracks are here microscopic and not much larger than the grain size, the metal behaves in these tests as quasibrittle). Note that, for such very short cracks, all the dimensions are infinitely larger than the crack length. Hence, the only size characteristic that exists is the crack length which must, therefore, be considered in the size effect law as the structure size.

For the present modified form, the fatigue threshold is obtained as

$$
\nu_{t h}=C\left(\Delta K_{t h}\right)^{m}\left(\frac{D+D_{0 m}}{D+D_{0 c}}\right)^{m / 2} \quad \text { or } \quad \frac{\Delta K_{t h}}{\Delta K_{0}}=\sqrt{\frac{D+D_{0 c}}{D+D_{0 m}}}
$$

As $D \rightarrow 0$, Eq. (33) would predict a constant $\Delta K_{t h}$. This does not agree with the data in [29], which closely follow Bažant's size effect law expected for quasibrittle materials. This is not surprising since even the classical Paris law cannot model the continuous transition from the crack growth under cyclic load, which is a function of $N$ (independent of time), to the crack growth under static (constant) load, which is a function of time $t$ and is described by the Charles-Evans law $[30 ; 31 ; 32 ; 33 ; 34]$.

Comment on Ramification to Metals: The fatigue of materials obeying LEFM is the limit case of fatigue of quasibrittle materials as the ratio of structure size to the FPZ (or RVE) size tends to infinity. Vice versa, the materials obeying LEFM become quasibrittle at a sufficiently small scale. Thus, for metals, in which the FPZ length is of micrometer scale, the fatigue of micrometer scale devices, such as, MEMS, must be expected to be similar to that of concrete structures, in which the FPZ length is about $0.5 \mathrm{~m}$. This similarity is worth exploring ${ }^{\mathrm{c}}$ _ especially given the evidence of quasi-brittleness in the structural strength of MEMS devices, see e.g. [35].

\section{Summary and conclusions}

1. The size effect experiments on fatigue in concrete verify that there is an appreciable size effect in the Paris law coefficient $C$. No noticeable size effect is seen in the exponent $m$.

2. The fatigue response of concrete and other quasibrittle materials suffers from a huge scatter in terms of lifetime, exceeding two orders of magnitude. This causes large scatter in the Paris law coefficient $C$, though not in the exponent $m$.

c1 Text added. 
3. Because of large scatter, general trends and the values of transitional size $D_{0 c}$ and ratio $R_{f}$ can be determined only from the averages of a number of repeated tests. Failing to do so can lead to misleading conclusions about the size of the cyclic FPZ.

4. The original form of the size adjusted Paris law (Eq. 7) does not yield $D_{0 c}$ values that are proportional to the cyclic FPZ size. This appears to be a shortcoming of this form.

5. The alternative form that is derived here (Eq. 19) yields $D_{0 c}$ values proportional to the cyclic FPZ size.

6. It is verified here, both experimentally and numerically, that the cyclic FPZ size is smaller than the monotonic one, its size being reduced to about $50 \%$ to $60 \%$.

7. Modeling shows that the cyclic FPZ size is not constant throughout the fatigue lifetime, but grows towards the end, approaching the monotonic FPZ size.

8. The average fatigue lifetime from experiments is seen to decrease with the size, consistent with predictions of the microplane model. Because of huge scatter in lifetimes, whether this is the true mean behavior could be verified by testing several specimens per size rather than only one.

9. In quasibrittle materials subjected to fatigue, with applied loads proportional to the structure strength, there is a strong size effect on the final crack length at failure.

10. Calculations using both the original and modified forms of the size adjusted Paris law show a non-monotonic size effect in fatigue lifetime. For smaller sizes the lifetime decreases with increasing size which is consistent with the experiments as well as modeling. Then the lifetime reaches a minimum, and afterwards it begins to increase. Eventually, for very large sizes, the lifetime size effect becomes linear and consistent with LEFM.

11. Same as the classical Paris law, the present form of the size-adjusted Paris law does not agree with the experimental data on crack length effect on fatigue threshold. Attaining agreement will require formulating a transition between the the Paris law for crack growth under cyclic loading and Charles-Evans law for crack growth under static loading.

Acknowledgment: Financial support by the U.S. National Science Foundation under Grant CMS-0556323 to Northwestern University is gratefully acknowledged. Thanks are due to Prof. Jia-Liang Le, Asst. Prof. at University of Minnesotta, for the many helpful discussions related to size effect in quasibrittle fatigue.

$\mathrm{c} 1$

\section{References}

[1] Z. P. Bažant, J. Planas, Fracture and Size Effect in Concrete and Other Quasibrittle materials, CRC Press, 1998.

[2] R. V. Pieri and G. B. Sinclair, On the effects of scaling on the Paris law, Int. J. Fracture, 68 (1994), R3-R8.

[3] J.M. Barsom, E.J. Imhof, S.T. Rolfe, Fatigue crack propagation in high yield strength steels, Engng Fracture Mech., 2 (1971), pp. 301317.

${ }^{\text {c1 }}$ Newly added references [2], [3], [4], [5], [6] $,[7],[8],[9],[35]$ 
[4] W. R. Brose and N. E. Dowling, Size effects on the fatigue crack growth rate of type 304 stainless steel, Elastic Plastic Fracture, ASTM STP 668, Philadelphia (1979) 720-735.

[5] W. G. Clarke Jr., Effect of Temperature and Section Size on Fracture Crack growth in vessel steel," Journal of Materials, 6-1 (1971), 134-149

[6] L. A. James, Specimen size considerations in fatigue crack growth rate testing, in Fatigue crack growth measurement and data analysis, ASTM STP 738, Philadelphia (1981), 45-57.

[7] S. K. Putatunda and J. M. Rigsbee, Effect of specimen size on fatigue crack growth rate in AISI 4340 steel, Engineering Fracture Mechanics, $22-2$ (1985), 335-345

[8] R. O. Ritchie, Incomplete self-similarity and fatigue crack growth, Int. J. Fracture, 132 (2005), 197-203.

[9] S. Ray and J. M. Chandra Kishen, Fatigue crack propagation model and size effect in concrete using dimensional analysis, Mechanics of materials, 43-2 (2011), 75-86.

[10] G. I. Barenblatt and L. R. Botvina, Incomplete self-similarity of fatigue in the linear range of crack growth, Fatigue of Engrg. Mater. and Struct., 3 (1981), 193-202.

[11] Z. P. Bažant, K. Xu, Size effect in fatigue fracture of concrete, ACI Mater. J., 88-4(1991), 390-399.

[12] Z. P. Bažant, W. F. Schell, Fatigue fracture of high-strength concrete and size effect, ACI materials journal, 90-5 (1993), 472-478.

[13] J. Le, J. Manning and J. Labuz, Scaling of fatigue crack growth in rock, Int. J. of Rock Mech. and Mining Sciences, 72 (2014) 71-79

[14] P. Paris, F. Erdogan, A critical analysis of crack propagation laws, J. Basic Engg., 85(1963), $528-534$.

[15] S. Suresh, Fatigue of materials, Cambridge: Cambridge University Press, 1991.

[16] K. Kirane and Z. P. Bazant, Enhanced microplane model for sub-critical crack growth and size effect under cyclic loading in isotropic quasibrittle solids, Int. J. Fatigue, 70 (2015), 93-105.

[17] Bažant, Z.P. 2005. Scaling of Structural Strength, 2nd ed. Elsevier, London.

[18] J. Rice, Mechanics of crack tip deformation and extension by fatigue, Am. Soc. forz Testing Materials, 415 (1967), 247-311.

[19] C. G. Hoover and Z. P. Bažant, Comprehensive concrete fracture tests: Size effects of Types 1 and 2, crack length effect and postpeak, Engrg. Fract. Mech., 110 (2013), 281-289.

[20] F. C. Caner, Z. P. Bažant, Microplane Model M7 for Plain Concrete I : Formulation, J. Eng. Mech. ASCE, 139 (2013), 1714-1723

[21] F. C. Caner, Z. P. Bažant, Microplane Model M7 for Plain Concrete II : Calibration and Verification, J. Eng. Mech. ASCE, 139 (2013), 1724-1735. 
[22] Z. P. Bažant, B. H. Oh, Microplane model for progressive fracture of concrete and rock, J. Eng. Mech., ASCE, 111 (1985), 559-582.

[23] Z. P. Bažant, B. H. Oh, Crack band theory for fracture of concrete, Matériaux et Construction, 16-3 (1983), 155- 177

[24] G. Cusatis, E. Schauffert, Cohesive crack analysis of size effect, Engineering Fracture Mechanics, 76 (2009), 2163-2173.

[25] Bažant, Z.P., Yu, Qiang., 2011, Size effect testing of cohesive fracture parameters and nonuniqueness of work-of-fracture method. ASCE J. of Engrg. Mech. 137 (8), 580-588.

[26] Bažant, Z.P., Le, J.-L., and Bazant, M.Z., "Scaling of strength and lifetime probability distributions of quasibrittle structures based on atomistic fracture mechanics", Proc. of the National Academy of Sciences 106 -28 (2009), 11484-11489.

[27] Le, J.-L., Bažant, Z.P., 2011. Unified nano-mechanics based probabilistic theory of quasibrittle and brittle structures: II. Fatigue crack growth, lifetime and scaling. J Mech Phys Solids 59, $1322-1337$.

[28] K. Kirane and Z. P. Bazant, Size effect in Paris law for quasibrittle materials analyzed by themicroplane constitutive model M7, Mech. Re. Commun. (2015) (in press)

[29] Tanaka, K., Nakai, Y., Yamashita, M., Fatigue growth threshold of small cracks. Int. J. Frac. 17 (1981), 519533

[30] Evans, A.G., A method for evaluating the time-dependent failure characteristics of brittle materials and its application to polycrystalline alumina J. Mater. Sci. 7 (1972), pp. 1137 1146

[31] Charles, R.J., Energy-size reduction relationships in comminution. Trans. Am. Inst. Min. Metall. Petrol. Engrs 208, 80-88.

[32] Charles, R.J. 1958a. Static fatigue of glass I. J. Appl. Phys. 29 (11): 1549-1553.

[33] Charles, R.J. 1958b. Static fatigue of glass II. J. Appl. Phys. 29 (11): 1554-1560.

[34] Hillig, W., B. and Charles, R., J., Surfaces, stress-dependent surface reaction, and strength, Proc.. 2nd Berkeley Int. Materials Conf., V.F. Zackay, ed. (1964).

[35] J. Le, R. Ballarini and Z. Zhu, Modeling of Probabilistic Failure of Polycrystalline Silicon MEMS Structures, J. American Ceramic Soc., 98-6 (2015), 1685-1697. 


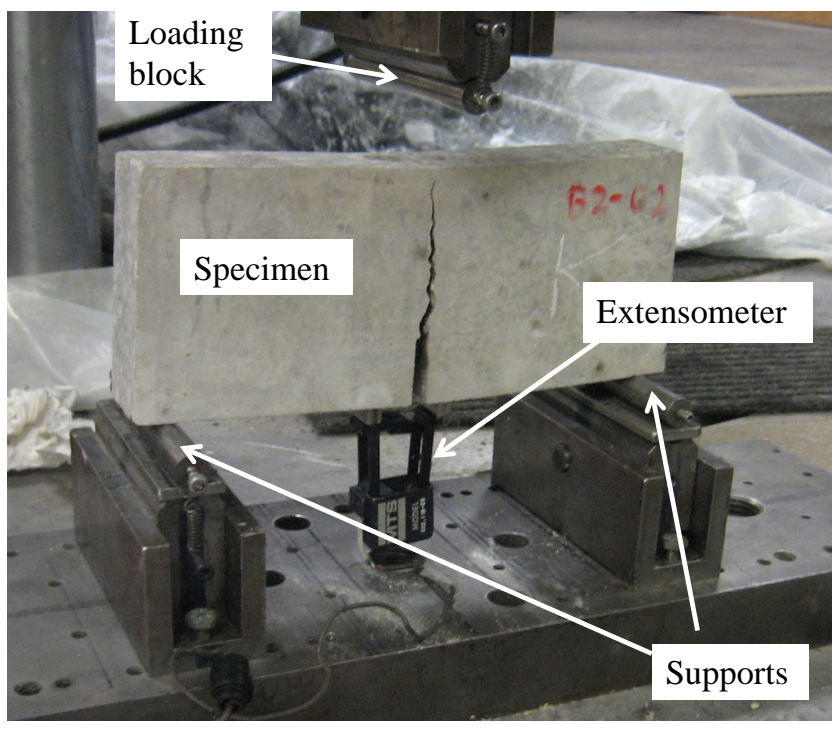

Figure 1: Medium size specimen with the fatigue test set up 


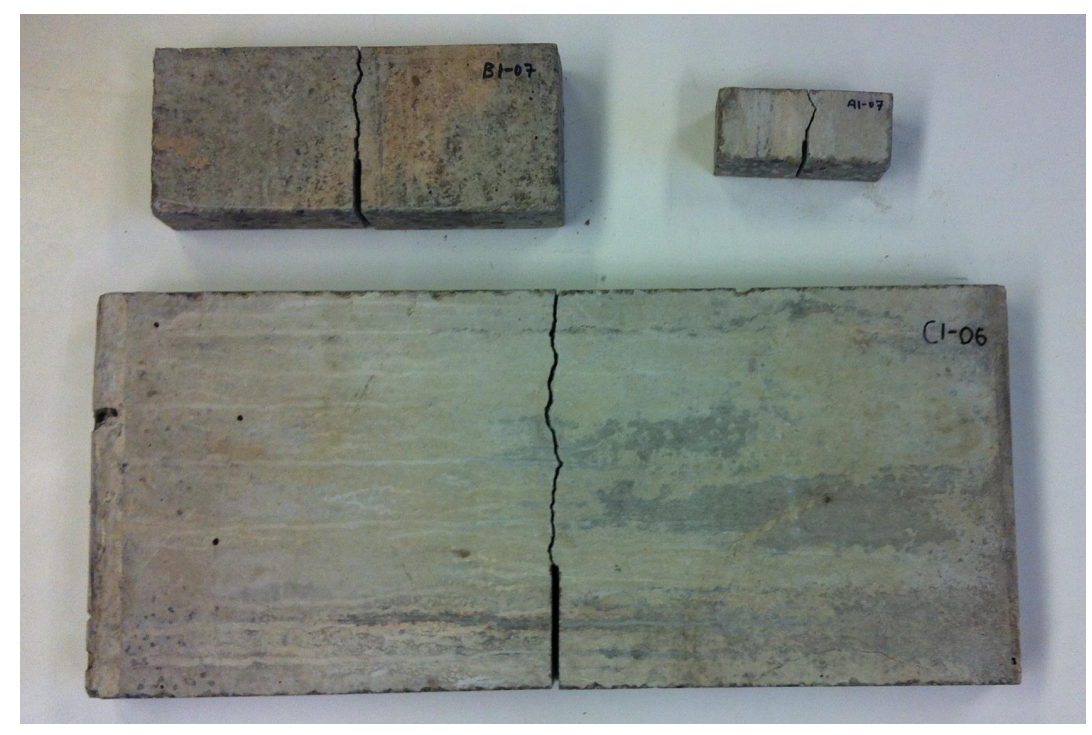

Figure 2: Specimen of each size after fatigue failure

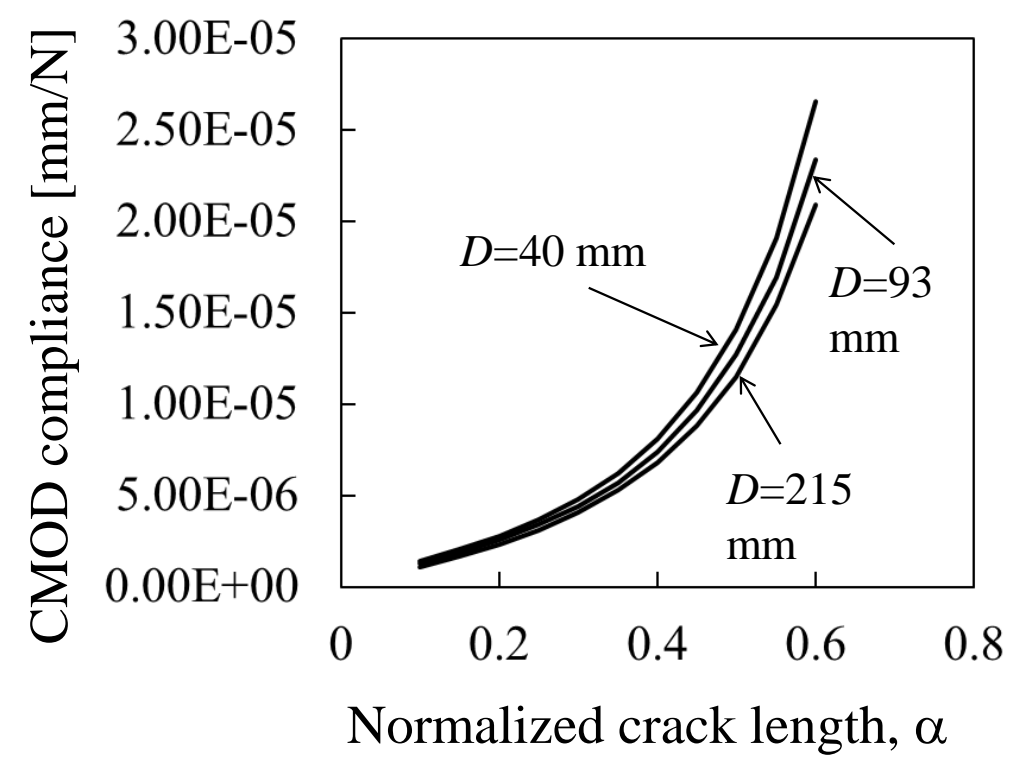

Figure 3: Compliance calibration curve for each size 


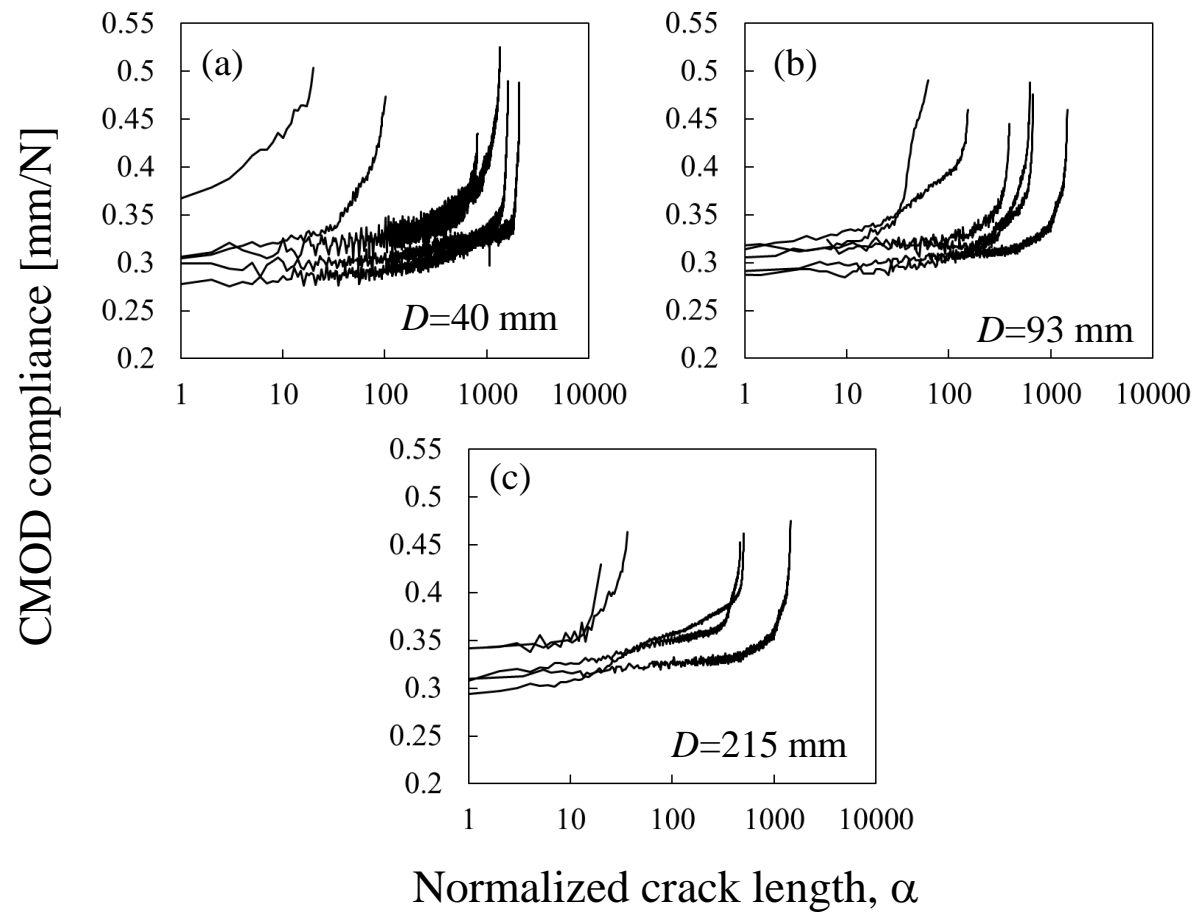

Figure 4: Evolution of normalized crack length (a) Small size specimen (b) Medium size specimen (c) Large size specimen 


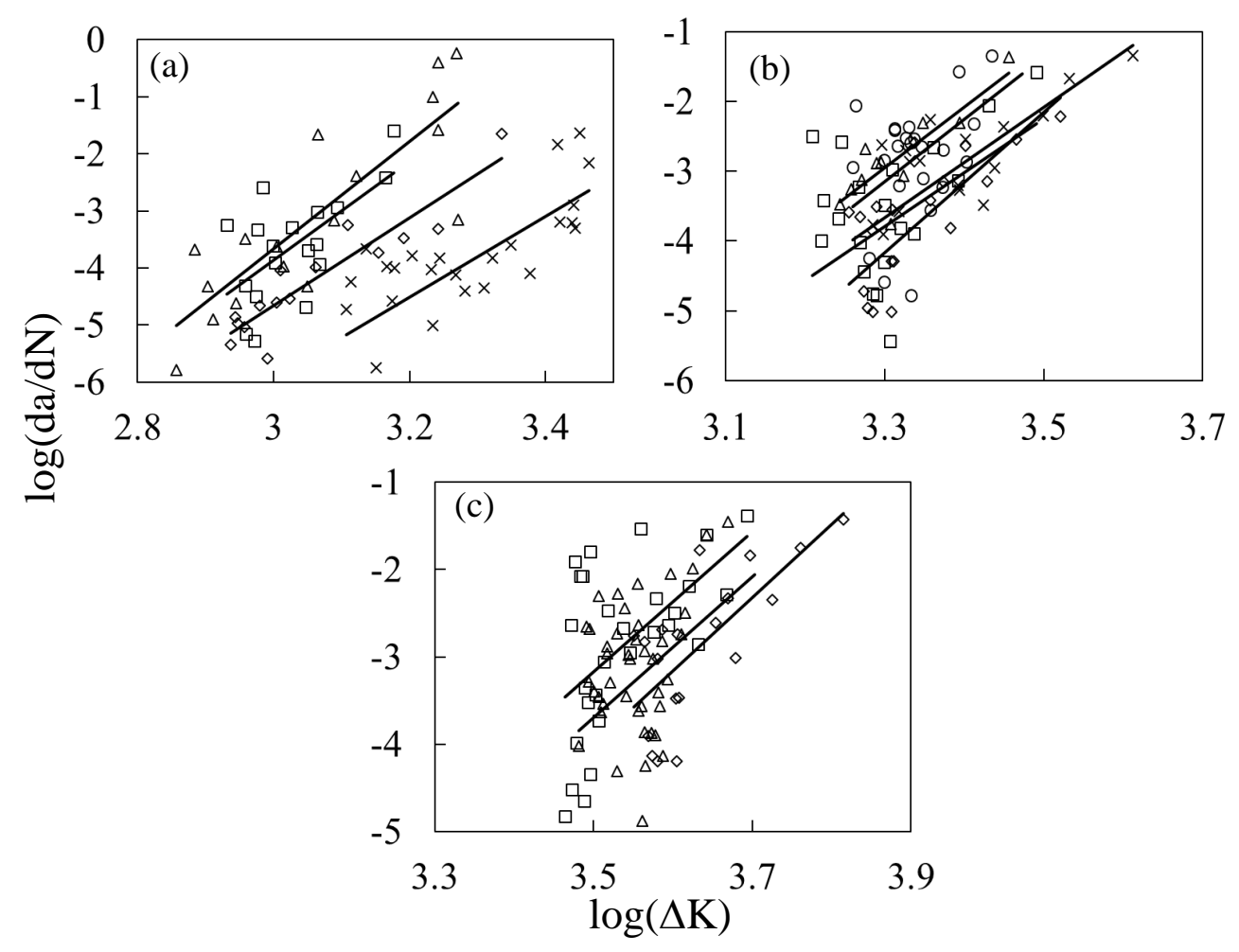

Figure 5: Paris law plots for individual specimen of (a) Small size (b) Medium size (c) Large size 

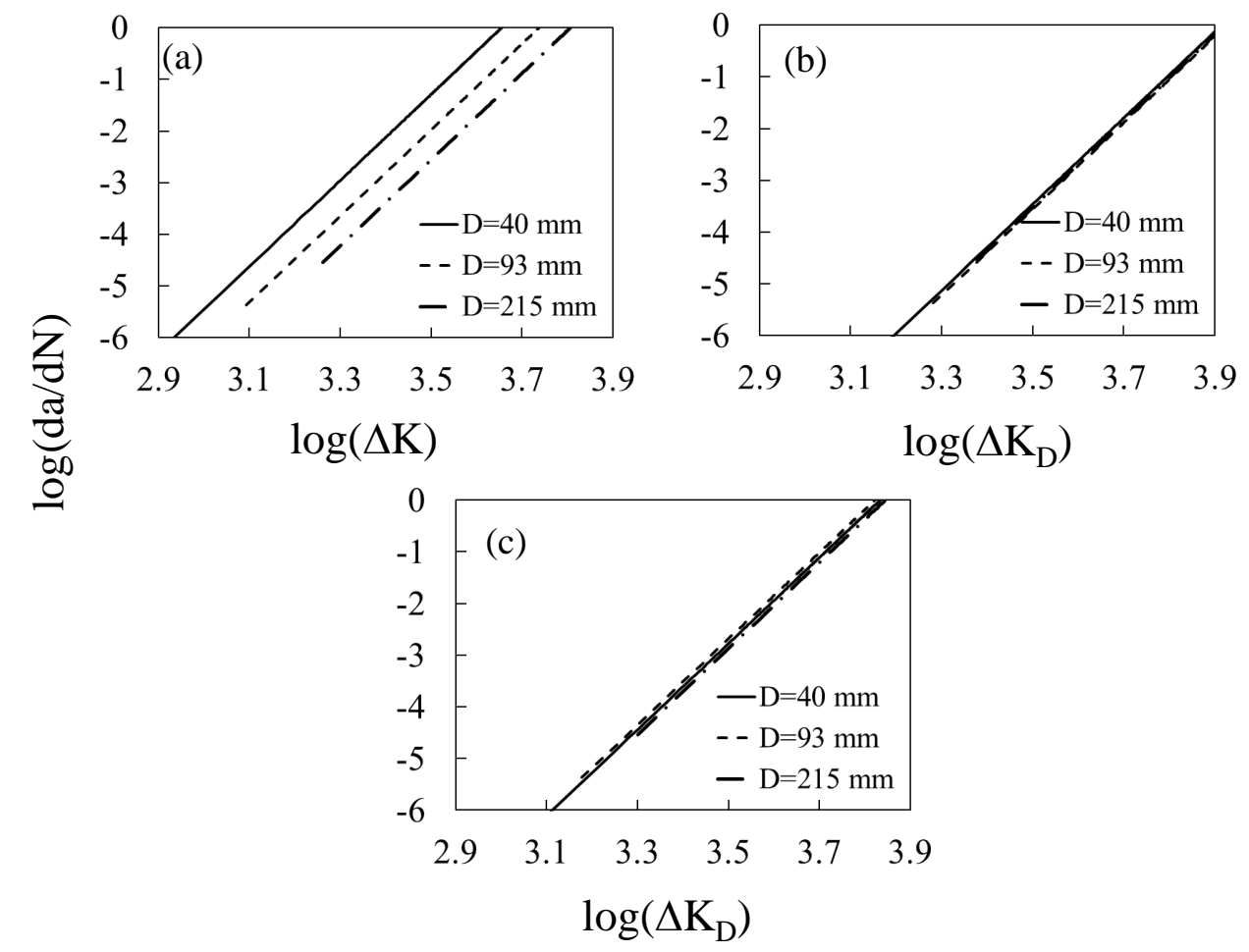

Figure 6: (a) Unadjusted Paris law plotted using average values, $\bar{C}$ and $\bar{m}$, for each size (b) Size adjusted Paris law, according to the original hypothesis (Eq. 7) (c) Size adjusted Paris law according to the modified hypothesis (Eq. 19 


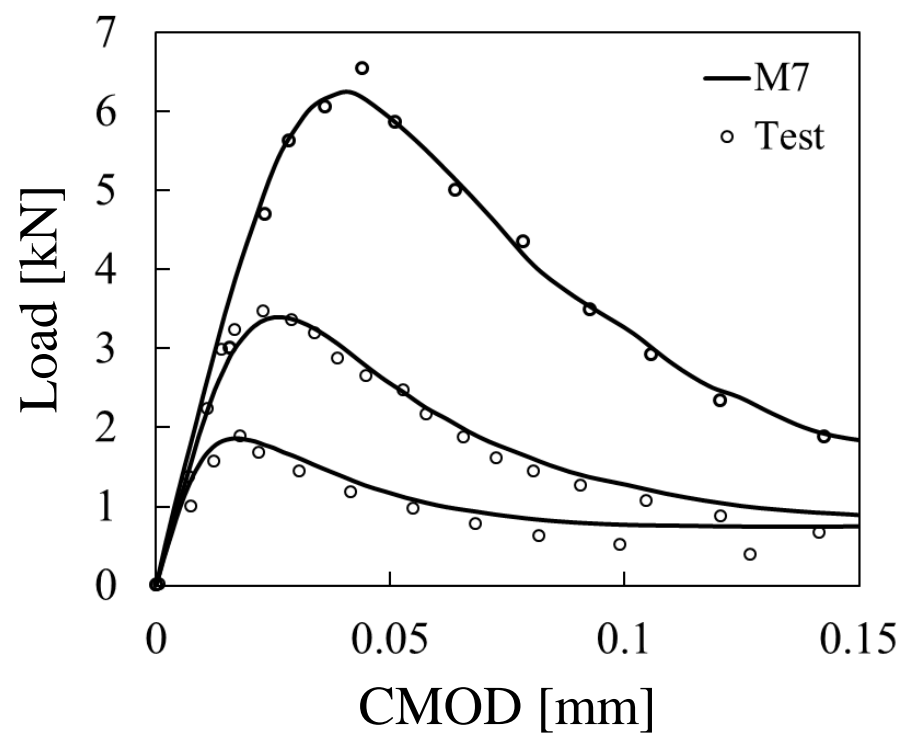

Figure 7: Comparison of monotonic response of beams from [19] predicted by $\mathrm{M} 7$ with experiments 


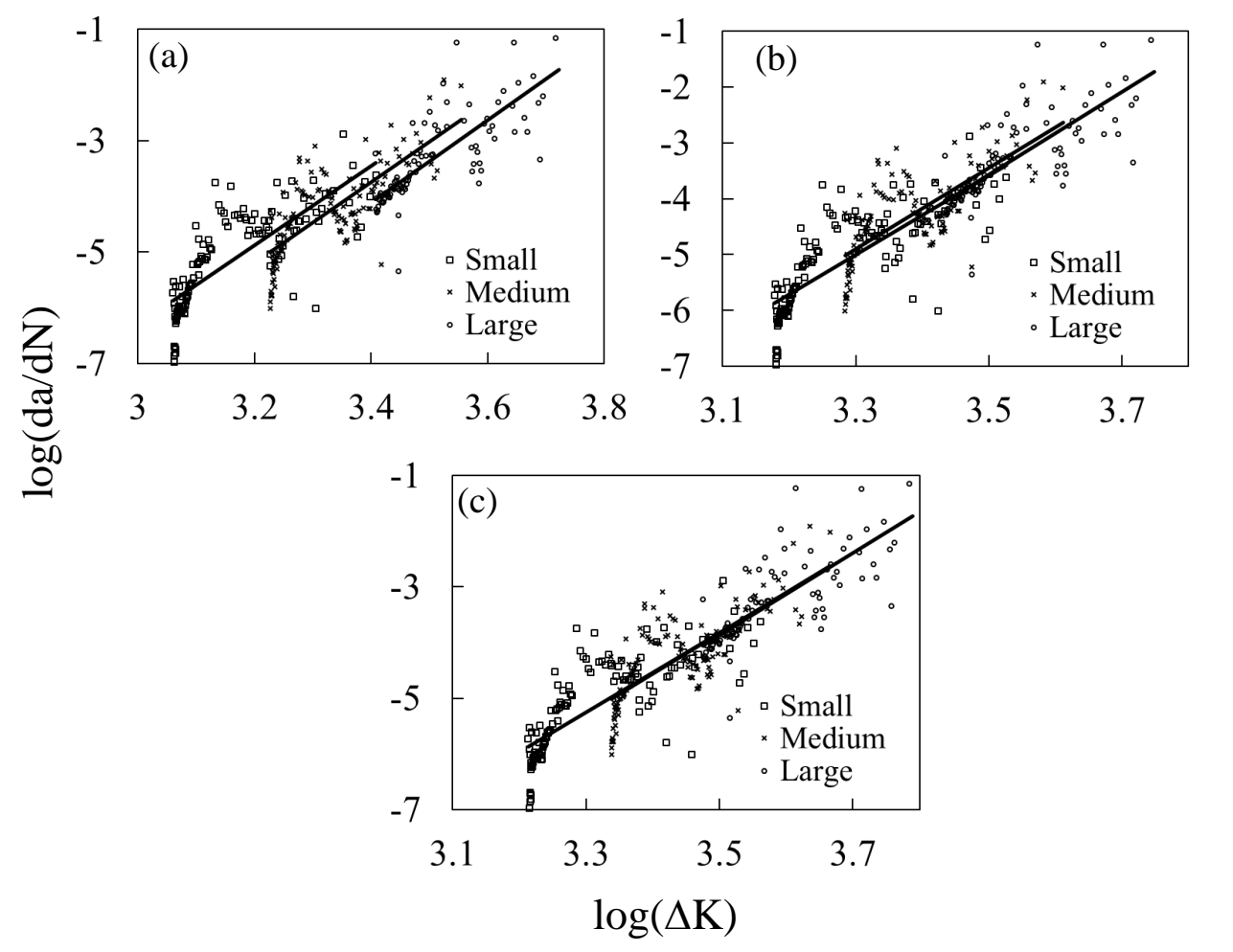

Figure 8: Predicted size effect in Paris law for concrete beams from [19] (a) Unadjusted plots, (b) Size adjusted plots according to the original hypothesis (Eq. 7), (c) Size adjusted plots - according to the modified hypothesis (Eq. 19)

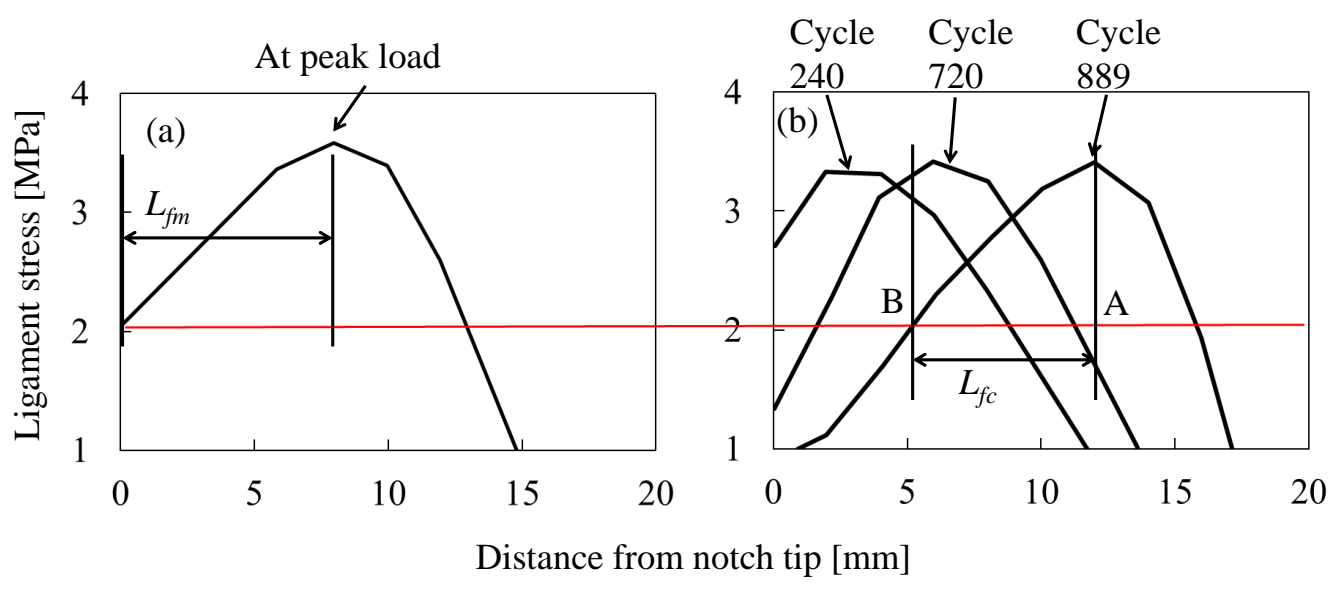

Figure 9: Measurement of FPZ size from the stress profile in the ligament for the small sized concrete beam (a) Monotonic (b) Cyclic 

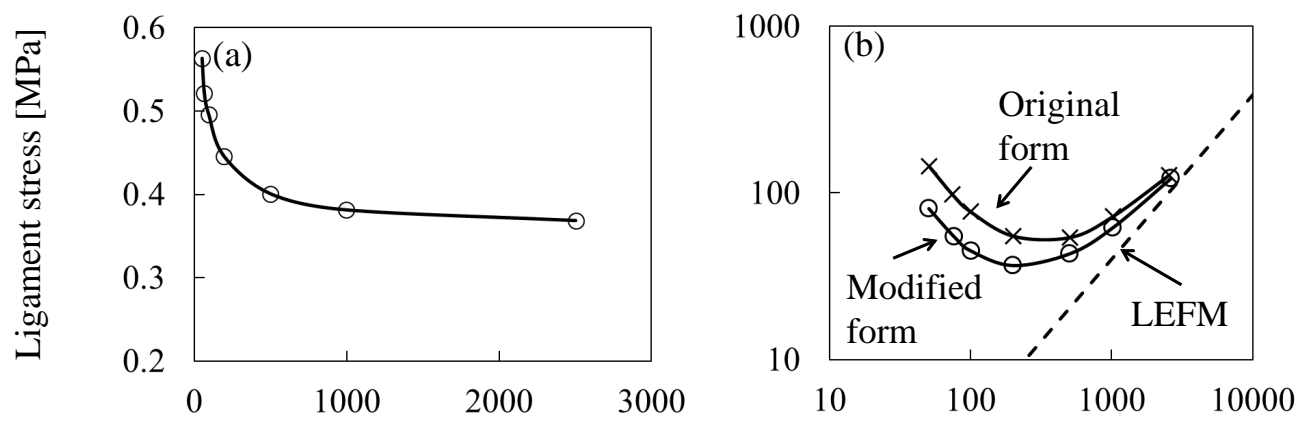

Distance from notch tip [mm]

Figure 10: (a) Calculated size effect in critical crack length at fatigue failure (b) Calculated size effect in fatigue lifetime, resulting from the original and modified forms of size adjusted Paris law 